\title{
Characterization of the specific cleavage of ceiE7-mRNA of the bactericidal ColE7 operon
}

\author{
Ssu-Jean Chang, ${ }^{\text {a }}$ Shih-Yang Hsieh, ${ }^{\mathrm{b}}$ Hanna S. Yuan, ${ }^{\mathrm{a}, \mathrm{b}}$ and Kin-Fu Chak ${ }^{\mathrm{a}, *}$ \\ ${ }^{a}$ Institute of Biochemistry, National Yang Ming University, Shih-Pai, Taipei 11221, Taiwan, ROC \\ ${ }^{\mathrm{b}}$ Institute of Molecular Biology, Academia Sinica, Taipei 11529, Taiwan, ROC
}

Received 23 October 2002

\begin{abstract}
Posttranscriptional control of the bactericidal ColE7 operon has been implicated by a feedback endonucleolytic cleavage of its own mRNA. The cleavage site has been located at the coding region of ceiE7, the second cistron of the ColE7 cea-cei-cel polycistronic transcript. Interestingly, $\operatorname{Im} 7$ protein, the translation product of $c e i E 7$, is required for the specific cleavage. It was found that both sequence (GAUCUGAUU) flanking the cleavage site and the putative T1 stem-loop structure distal to the coding region of $c e i E 7$ gene play a critical role for the specific cleavage of $c e i E 7-m R N A$. Furthermore, we have verified that a di-nucleotide GG sequence located at the topmost position of the loop region of the putative stem-loop structure is essential for the specific cleavage of ceiE7-mRNA. Thus, our data reveal the existence of a novel mRNA degradative machinery for the regulation of the expression of ColE7 operon.

(c) 2002 Elsevier Science (USA). All rights reserved.
\end{abstract}

Keywords: Colicin E7; mRNA decay; Specific cleavage of mRNA; Stem-loop structure of RNA

The ColE operon comprises the colicin structural (cea), immunity (cei), and lysis genes (cel). Bactericidal activities of colicins have been classified as DNase (colicin E2, E7, E8, and E9), RNase (colicin E3, E6, and cloacin DF13), ionophore (colicin E1, A, N, K, Ia, and B), inhibition of protein synthesis (colicin E5 and D), and inhibition of synthesis of murein and LPS (colicin M) [1,2]. Immunity protein (Im) encoded by the cei gene is host's antidote for colicin [3-5]. Lys protein (or bacteriocin-release protein, $\mathrm{BRP}$ ), the $c e l$ gene product, activates phospholipase A in the outer membrane of Escherichia coli cells and consequently alters the permeability of the outer membrane and facilitates colicin release from the periplasm [6]. Overexpression of cea and cel is lethal to the host; therefore, a tightly controlled SOS promoter is utilized to safeguard the host cells containing the ColE operon [7].

We recently reported that immunity protein $(\operatorname{Im} 7)$ of colicin E7 mediates a site-specific cleavage of its own mRNA [8]. The crystal structures of monomeric [9] and

\footnotetext{
${ }^{*}$ Corresponding author. Fax: +886-2-28264843.

E-mail address: kfchak@ym.edu.tw (K.-F. Chak).
}

dimeric forms [8] of $\operatorname{Im} 7$ protein have been resolved. Most interestingly, it was found that the monomeric form of Im7 is a DNase inhibitor, while the dimeric form may perform a putative novel RNase activity. Thus a novel autoregulatory expression of the $c e a-c e i-c e l$ polycistronic transcript of the ColE7 operon has been proposed [8].

In prokaryotes, the rate-limiting step in mRNA decay is usually an initial endonucleolytic cleavage, followed by rapid $3^{\prime}-5^{\prime}$ exoribonuclease degradation [10-12]. Two endoribonucleases, RNase III and RNase E [13,14], are largely responsible for bulk mRNA processing at either the $5^{\prime}$ untranslated region (UTR) [15-17] or $3^{\prime}$ UTR [18], while RNase II and polynucleotide phosphorylase [19] are mainly responsible for bulk mRNA degradation in a fashion of $3^{\prime}-5^{\prime}$ direction into mono-nucleotides. It has been shown that the consensus sequence at the cleavage site of RNase $\mathrm{E}$ is (G/A)AUU(A/U) [16,20]. Moreover, the 9S rRNA and S20 mRNA structures in RNase E cleavage reveal that secondary structure adjacent to the sites of cleavage contributes to the site selection for the specific cleavage by RNase E [21,22]. In contrast, the cleavage site of RNase E in RNA I of pBR322 indicates 
that the RNase $\mathrm{E}$ is a single-strand-specific enzyme with preferential hydrolysis at $\mathrm{N}^{\boldsymbol{\nabla}}$ AU sequence [23,24].

So far, none of the available endoribonucleases that we have tested is responsible for the specific cleavage of ceiE7-mRNA. In this report, we further characterized that the sequence flanking the specific cleavage site and the secondary structure in the $3^{\prime}$ UTR of cei-mRNA are important for this specific cleavage. The exact role of $\operatorname{Im} 7$ in this cleavage is still unanswered. However, based on our experimental results, we propose that $\operatorname{Im} 7$ might act as a mediator to recruit some unknown factors binding to ceiE7-mRNA for initiating the specific cleavage of its own mRNA.

\section{Materials and methods}

Bacterial strains, plasmids, and growth conditions. Escherichia coli W3110 containing plasmid ColE7-K317 has been previously characterized [25]. E. coli JM101, M15, and JM109, were the bacterial hosts for recombinant plasmids constructed in this study. Plasmids, pGEM3Zf(+), pUC18, and pREP4, were used as cloning vectors. Plasmid pYAN13R [8] containing the ceiE7 gene was used as template for the preparation of run-off ceiE7 transcripts. E. coli cells were cultured in LB with vigorous shaking [26]. For cloning vector selection, ampicillin was added to a final concentration of $100 \mu \mathrm{g} / \mathrm{ml}$; for induction of the cloned genes, IPTG or mitomycin C (MMC) was added to final concentrations of $1 \mathrm{mM}$ and $0.5 \mu \mathrm{g} / \mathrm{ml}$, respectively.

Construction of expression vectors. Positions of mutated nucleotides are indicated by their relative distances to the major cleavage site of the ceiE7-mRNA. Thus, a nucleotide upstream and downstream of the major cleavage site is designated as -1 and +1 , respectively.

For PCR-mediated site-directed mutagenesis, plasmid ColE7-K317 containing a complete ColE7 operon [25] was used as template for polymerase chain reaction (PCR) amplification. In the construction of pSC02, a 200-bp DNA fragment containing the regulatory region of ColE7 operon [7] was amplified by PCR using a pair of primers: Pcoll (5'CAACGCCTGCAGGACCGCGAAAGG- $3^{\prime}$ ) and Pcol2 (5'-ATTC CCTCTAGAAACGTTAAAAC- $\left.3^{\prime}\right)$. $P s t \mathrm{I}$ and $X b a \mathrm{I}$ sites were generated at either ends of the PCR-amplified fragment (represented in bold within primer sequence). Similarly, another 387-bp DNA fragment containing the entire $c e i E 7$ gene was amplified by PCR using a pair of primers: Pim 1 (5'-GATATTTCTAGAGGTAAATAATATGG-3') and Pim2 (5'-CT GCAAGAATTCATAAAATAATCCC- $\left.3^{\prime}\right)$. In this fragment, $X b a \mathrm{I}$ and EcoRI sites were generated at either ends of the PCR-amplified fragment. The two PCR-amplified fragments were digested with restriction enzymes and subsequently ligated with each other through $X b a$ I site. The resultant Pst $\mathrm{I} / E \operatorname{coRI}$ DNA fragment containing the "SOS" response promoter and the entire $\mathrm{ceiE7}$ gene of the ColE7 operon was cloned into the $P s t \mathrm{I}$ and $E c o$ RI sites of the pUC18 vector. The resulting recombinant plasmid designated as pSC02 was transformed into E. coli JM101 (Fig. 1A). With pSC02 as template, the PCR-mediated site-specific mutagenesis was employed to generate mutations, as previously described [7]. Four types of mutations have been constructed: type I, the frame-shift mutations of the $c e i E 7$ gene including pSC02-16 and pSC02-28 (Fig. 1B); type II, nucleotide substitution mutants including pSC02-5, pSC02-12, pSC02-24, and pSC012-13 (Fig. 3A); type III, deletion mutants including pSC02-14R and pSJ-I1 (Fig. 4A); and type IV, mutations of the putative T1 stem-loop structure including pSC02-10, pSC02-25, and pSC02-26 (Fig. 5A). Primers for the plasmid constructions used in this work are listed in Table 1.

Extraction of total RNA and primer extension. Total RNA was extracted from lysed cells by the hot phenol method as described by

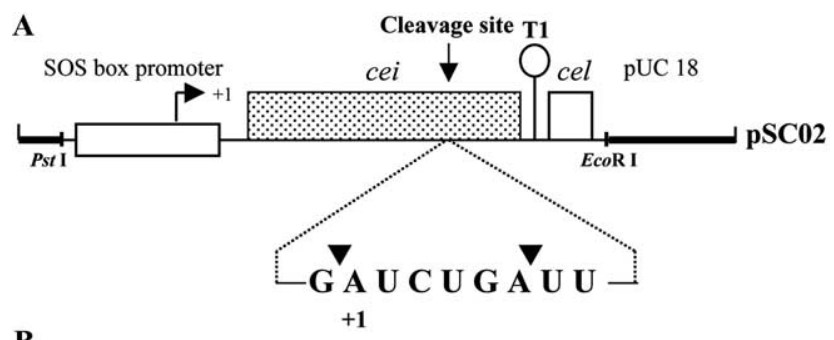

B

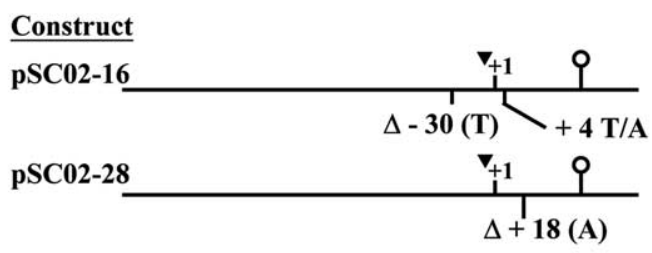

C

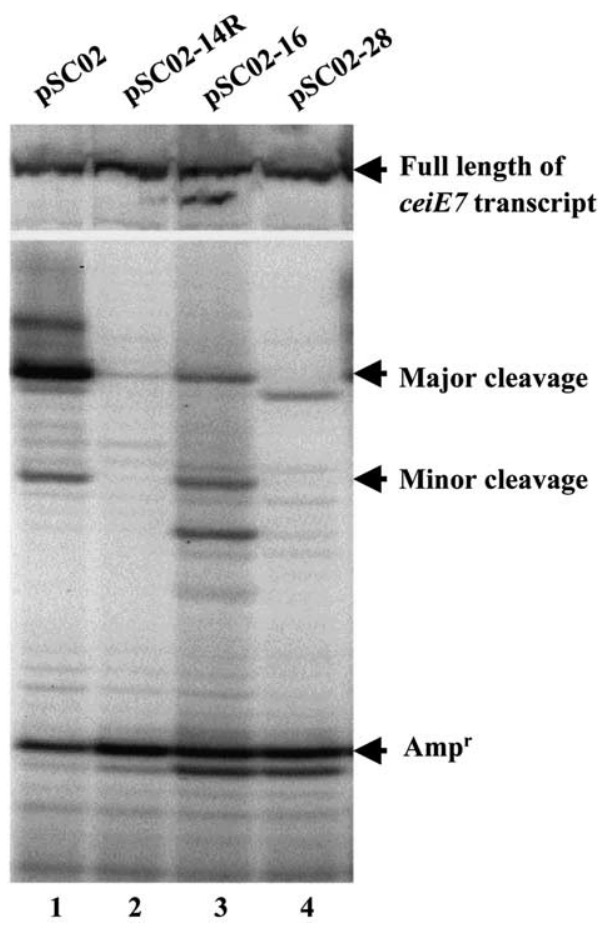

Fig. 1. Importance of the endogenously expressed $\operatorname{Im} 7$ protein for the specific cleavage of ceiE7-mRNA. (A) A schematic diagram of recombinant pSC02 plasmid used in this study. The arrows denote the major $\left(G^{\boldsymbol{\nabla}} \mathrm{A}\right)$ and minor $\left(\mathrm{A}^{\boldsymbol{\nabla}} \mathrm{U}\right)$ cleavage sites, respectively. The recognition sequence for the specific cleavage of ceiE7-mRNA is also shown. (B) Two out-of-frame mutants of the ceiE7 gene have been constructed to elucidate the importance of the functional $\operatorname{Im} 7$ protein for the specific cleavage of ceiE7-mRNA. Detailed construction of pSC02-16 and pSC02-28 was described in the text. The relative positions of the mutant sequences with respect to the major cleavage site are indicated. (C) Equal amounts of total RNAs $(50 \mu \mathrm{g})$ from cells containing pSC02-16 and pSC02-28 were analyzed by primer extension using reverse transcriptase and two $5^{\prime}$ end-labeled DNA primers, Pcei and $\mathrm{Pamp}^{\mathrm{r}}$, which are complementary to the ceiE7 coding region and the $a m p^{r}$, respectively. Lane 1, RNA sample from cells containing pSC02 as positive control; lane 2, RNA sample from cells containing pSC02-14R, a deletion mutant of cei gene, as negative control; lanes 3 and 4, samples from the two out-of-frame mutants, pSC02-16 and pSC02-28, respectively. It is noted that equal amount of RNA samples $(50 \mu \mathrm{g})$ was used for each primer extension experiment. Expression of plasmid-borne $a m p^{r}$ gene was detected and used as the internal control. 
Table 1

Oligonucleotide primer sequences for the construction of expression vectors, pSC02 and its derivatives

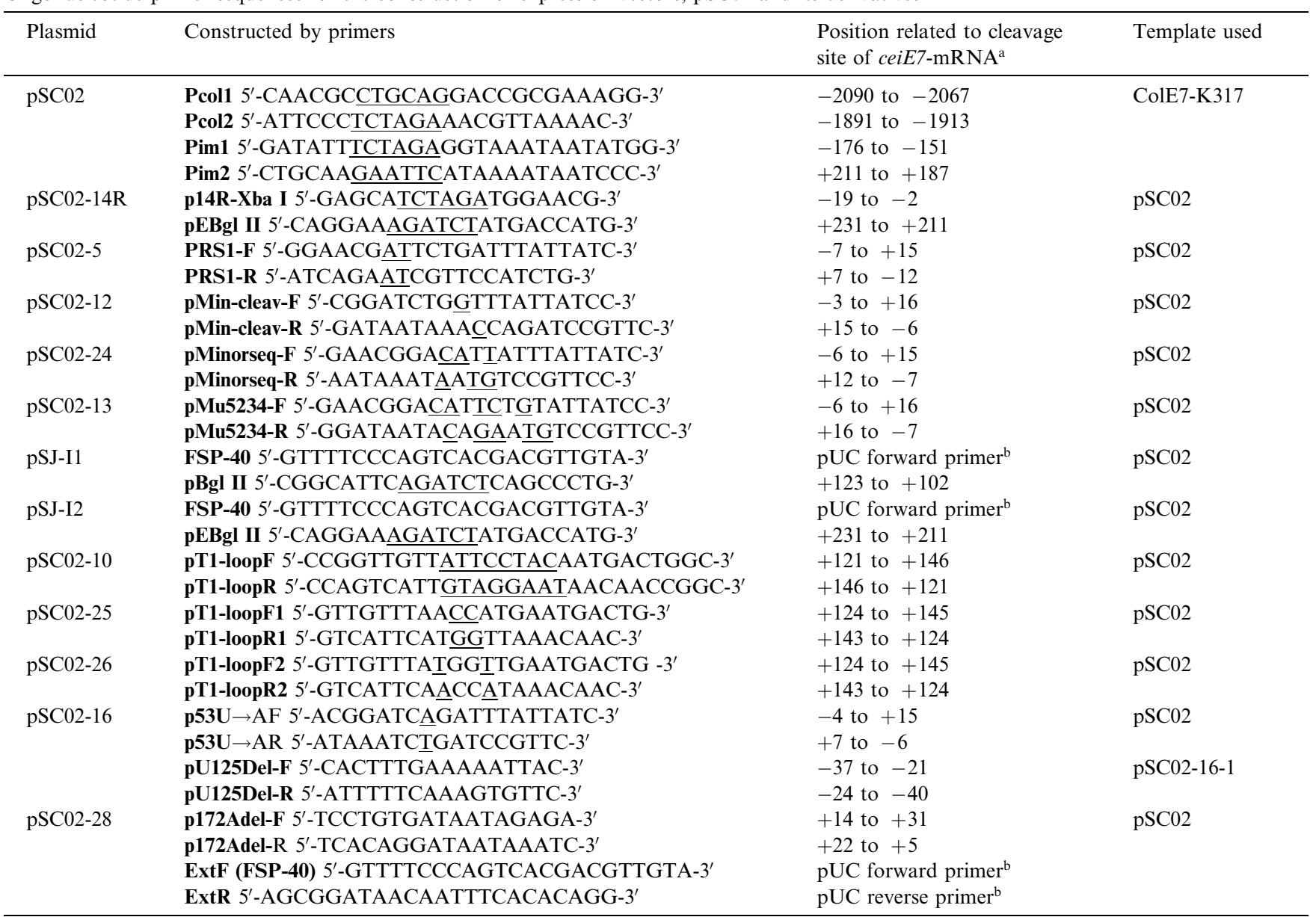

Note. The mismatched bases are underlined.

${ }^{\text {a }}$ All nucleotide positions are indicated relative to the major cleavage site of $c e i E 7-m R N A$; the nucleotide upstream of the cleavage site is -1 and downstream is +1 .

${ }^{\mathrm{b}}$ The universal primers flank on both sides of the multiple cloning site of pUC18.

von Gabain et al. [27]. For induction, bacteria were grown to an $\mathrm{OD}_{600}$ of 0.6 in L broth before addition of MMC $(0.5 \mu \mathrm{g} / \mathrm{ml})$ or IPTG $(1 \mathrm{mM})$. Samples were withdrawn at different time points and the total RNAs were extracted and analyzed by primer extension. All other details were the same as previously described [8].

Primer extension was carried out as the method described by Sambrook et al. [26]. Oligonucleotide primers used in the primer extension analysis were as follows: Pcei, 5'-TGGCTTACCGTTAGCAG (complementary to nucleotides $5^{\prime}+92 /+763^{\prime}, \approx 92 \mathrm{nt}$ downstream of the major cleavage site) and $\mathrm{Pamp}^{\mathrm{r}}, 5^{\prime}$-AGGGAATAAGGGCGACACGG (used for the primer extension to detect the expression level of ampicillin gene of the vector, the product of which can be used as internal control of the experiment).

In vitro transcription and preparation of $S$-30 cell free extracts from E. coli. The SP6 promoter fused with a full-length ceiE7 was amplified by PCR using a forward primer Psp6 (5'-ATTTAGGTGACACTATA G-3') and a reverse primer Pcel (5'-CATAACGACTCCTTGTTG- $3^{\prime}$ ). The PCR fragment amplified from pYAN13R was then used as template for in vitro transcription using SP6 RNA polymerase (Promega) according to the instructions from the manufacturer. The RNA was resuspended in an appropriate amount of RNase-free water.

Preparation of S-30 cell free extracts was the same as described by Mackie [28] except that the cell culture was incubated at $37^{\circ} \mathrm{C}$ instead of $30^{\circ} \mathrm{C}$.
In vitro ribonuclease activity assay. In vitro ribonuclease activity assay of the $\operatorname{Im} 7$ protein together with S-30 cell free extracts was carried out as previously described by Mackie [28].

Western blotting. For detection of $\operatorname{Im} 7, \mathrm{~S}-30$ cell free extracts were run in SDS-PAGE using a Mighty Small apparatus with a $12.5 \%$ running gel and a $5 \%$ stacking gel. Proteins were then blotted onto a PVDF membrane by using semidry apparatus (American Bionetics). After blocking, the membrane was hybridized with polyclonal antibodies against $\operatorname{Im} 7$ followed by anti-IgG antibodies conjugated with horseradish peroxidase. Protein bands that interacted with antibodies were visualized by treating the membrane with NEN, Western blot chemiluminescence reagent plus. The detailed procedure for hybridization was the same as that described in the NEN Western blot manuscript.

\section{Results}

Importance of the $\operatorname{Im} 7$ protein for the specific cleavage of ceiE7-mRNA in vivo

We have reported that $\operatorname{Im} 7$ protein is required for the specific cleavage of the ceiE7-mRNA in vitro [8]. To 
elucidate the importance of $\operatorname{Im} 7$ protein involving in the specific cleavage of the ceiE7-mRNA in vivo, using pSC02 as template (Fig. 1A), two frame-shift mutations (pSC02-16 and pSC02-28, Fig. 1B) of the ceiE7 gene were constructed. The cloned ceiE7 gene in pSC02-16 has a $\mathrm{T}$ to A transversion mutation at the " +4 " position

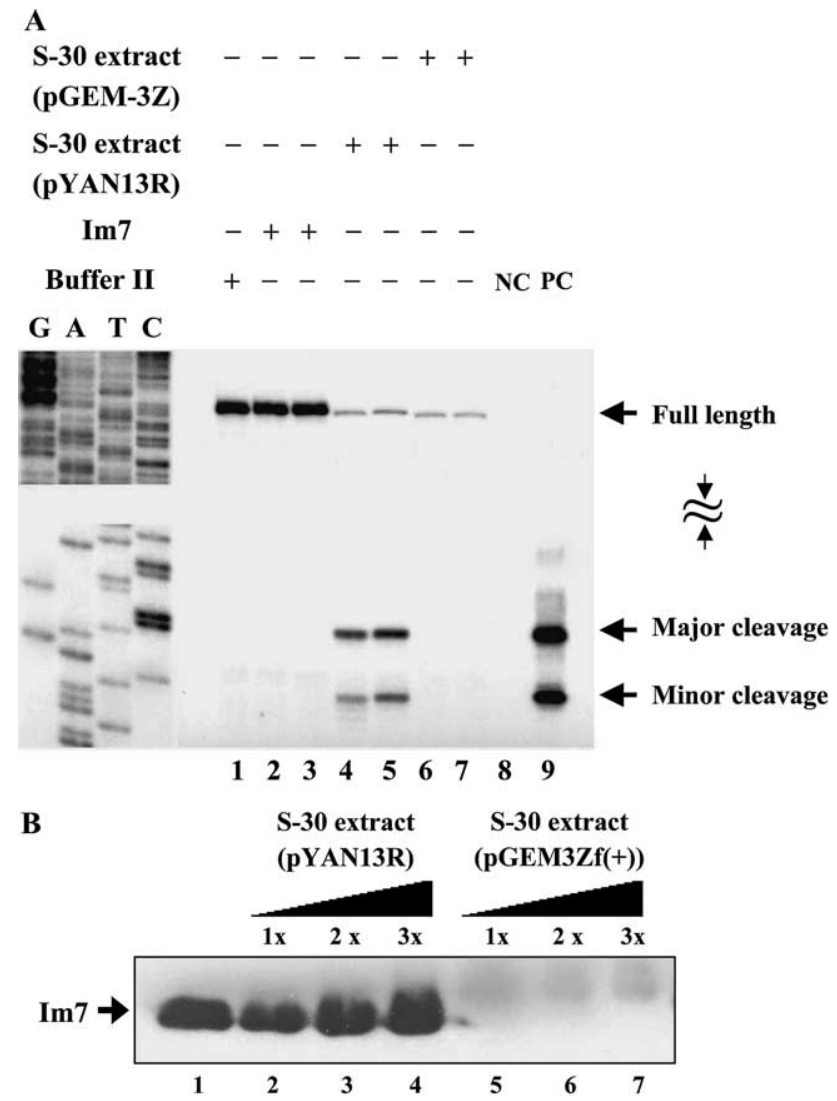

Fig. 2. In vitro ribonucleolytic activity assay for the specific cleavage of ceiE7-mRNA. In (A), a $500 \mathrm{ng}$ SP6 promoter-transcribed ceiE7mRNA (438 bp) was used for each reaction. Resultant mixtures were deproteinized, precipitated with ethanol, and then followed by primer extension analysis (see Materials and methods). These cDNA products were resolved by $6 \% \mathrm{w} / \mathrm{v}$ polyacrylamide sequencing gel and visualized by autoradiography. Primer Pcei was used both for primer extension and nucleotide sequencing as shown in the left-hand panel. Lane 1, RNAs were incubated with buffer II ( $50 \mathrm{mM}$ Tris- $\mathrm{HCl}, \mathrm{pH} 7.6,10 \mathrm{mM}$ $\mathrm{MgCl}_{2}, 60 \mathrm{mM} \mathrm{NH}_{4} \mathrm{Cl}, 0.5 \mathrm{mM}$ EDTA, $0.2 \mathrm{mM}$ PMSF, $0.1 \mathrm{mM}$ DTT, and $5 \%$ glycerol) at $37^{\circ} \mathrm{C}$ for $10 \mathrm{~min}$; lanes 2 and 3, with purified $\mathrm{Im} 7$ $(2.8 \mu \mathrm{g})$; lanes 4 and 5 , with $\mathrm{S}-30$ extracts (containing $14 \mu \mathrm{g}$ proteins) from E. coli JM109[pYAN13R(ceiE7)]; lanes 6 and 7, with S-30 extracts from $E$. coli JM109[pGEM3Zf(+)]; lanes 8 and 9, primer extensions of total RNAs $(30 \mu \mathrm{g})$ from $E$. coli JM109 [pGEM3Zf(+)] and E. coli JM109[pYAN13R], respectively. For experiments shown in lanes 2,4 , and 6 , samples were incubated at $37^{\circ} \mathrm{C}$ for $5 \mathrm{~min}$; and the duplicated samples in lanes 3,5 , and 7 were incubated for $10 \mathrm{~min}$. Only the top and bottom parts of the gel are shown. (B) Detection of endogenously expressed $\operatorname{Im} 7$ in S-30 extracts using in this experiment by Western blotting with antiserum against $\operatorname{Im} 7$. Lane 1, purified $\operatorname{Im} 7$, as positive control; lanes $2-4$, one- to threefold increasing amounts of S-30 extracts (containing $5-15 \mu \mathrm{g}$ proteins) from cells harboring pYAN13R; lanes 5-7, S-30 extracts from cells harboring pGEM$3 Z f(+)$. (meaning the fourth nucleotide downstream of the major cleavage site) and a nucleotide deletion at the " -30 " position (meaning the thirtieth nucleotide upstream of the major cleavage site); the cloned ceiE7 gene in pSC0228 has a nucleotide A deleted at the " +18 " position (Fig. 1B). Both frame-shift mutants are unable to express Im7 protein (data not shown). Total RNA extracts from cells harboring the mutants, pSC02-16 and pSC02-28, were analyzed by primer extension. Results of primer extension showed that in the absence of functional $\operatorname{Im} 7$ protein, the mutant ceiE7 mRNAs displayed a very weak specific cleavage signal (Fig. 1C, lanes 3 and 4). Thus, it was concluded that the functional $\operatorname{Im} 7$ protein is important for this specific cleavage in vivo.

\section{Specific cleavage of the ceiE7-mRNA in vitro}

The importance of $\operatorname{Im} 7$ for the specific cleavage of ceiE7-mRNA was further characterized by in vitro ribonucleolytic activity assay. As shown in Fig. 2A, the specific cleavage of ceiE7-mRNA occurred only in the presence of S-30 cell free extracts from $E$. coli containing the $\operatorname{Im} 7$ protein (Fig. 2A, lanes 4 and 5). Neither $\operatorname{Im} 7$ (Fig. 2A, lanes 2 and 3) nor S-30 cell free extracts alone (Fig. 2A, lanes 6 and 7) exerted ribonucleolytic activity for the specific cleavage of ceiE7-mRNA. It is worth noting that only a trace amount of the full-length extension product was detected in the in vitro assay with S-30 cell free extracts from cells containing no $\operatorname{Im} 7$ protein (Fig. 2A, lanes 6 and 7). It seems that the ceiE7mRNA was degraded rather rapidly in these S-30 cell free extracts. Western blot analysis confirmed that $\operatorname{Im} 7$ protein is found only in S-30 cell free extracts from E. coli containing the pYAN13R (Fig. 2B, lanes 2-4).

Thus, the in vitro ribonucleolytic activity experiments implied that in addition to $\operatorname{Im} 7$ protein, some factors from S-30 cell free extracts are also crucial for the determination of the specific cleavage of ceiE7-mRNA. This finding is different from the previous report that the $\operatorname{Im} 7$ protein alone can mediate the specific cleavage of the ceiE7-mRNA [8]. We speculate that the discrepancy between these two observations might be due to the contamination of cell-extracts in the purified $\operatorname{Im} 7$ protein of the previous study. Furthermore, it seemed that the $\operatorname{Im} 7$ protein might have an additional function either directly or indirectly to stabilize the ceiE7-mRNA from degradation in this assay system.

Characterization of the recognition sequence for the specific cleavage of ceiE7-mRNA

A major specific cleavage site of ceiE7-mRNA has been mapped between $\mathrm{G}$ and A nucleotides at the Asp52 codon (GAU) of the ceiE7 gene. Also, a concomitant minor specific cleavage site appeared between $\mathrm{A}$ and $\mathrm{U}$ nucleotides at the Ile54 codon (Fig. 3). To characterize 
A

\begin{tabular}{|c|c|c|c|}
\hline Construct & Flanking sequences of cleavage site & \multicolumn{2}{|c|}{ Cleavage signa } \\
\hline & +1 & Major & $\underline{\text { Minor }}$ \\
\hline pSC02 & 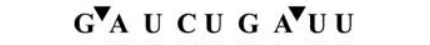 & +++ & + \\
\hline pSC02-5 & $\underline{A} \mathbf{U} U \mathbf{C} U \mathbf{G} A \mathbf{U} \mathbf{U}$ & + & ++ \\
\hline pSC02-12 & $\mathbf{G} A \mathbf{U} \mathbf{C} \mathbf{U} \mathbf{G} \underline{\mathbf{G}} \mathbf{U} \mathbf{U}$ & - & ++ \\
\hline pSC02-24 & $\mathbf{G} \mathbf{A} \underline{\mathbf{C}} \quad \mathbf{A} \mathbf{U} \underline{\mathbf{U}} \mathbf{A} \mathbf{U} \mathbf{U}$ & - & - \\
\hline pSC02-13 & $\mathbf{G} \mathbf{A} \underline{\mathbf{C} A} \mathbf{U} \underline{\mathbf{U}} \mathbf{C} U \underline{\mathbf{G}}$ & - & - \\
\hline
\end{tabular}

B

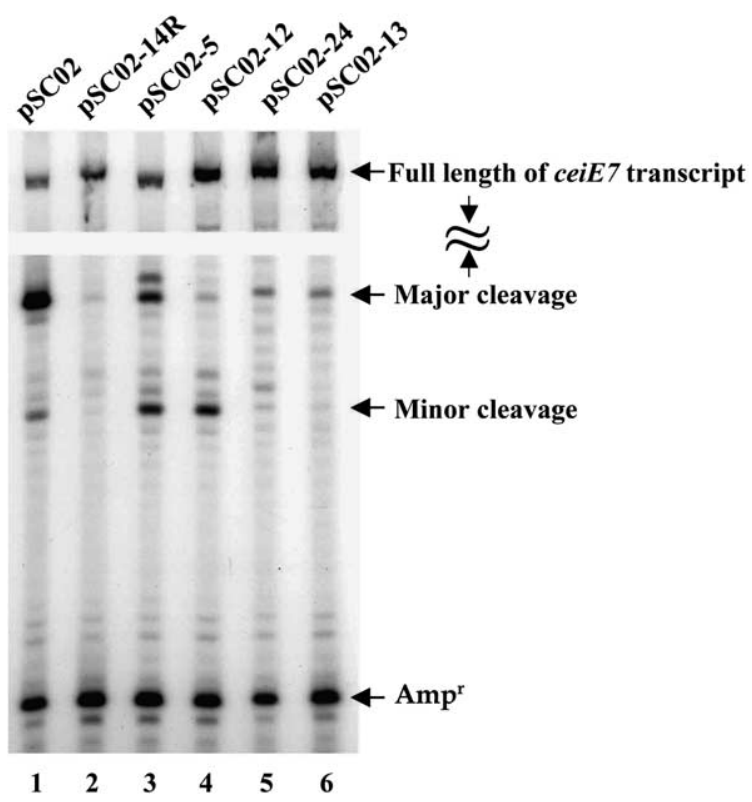

Fig. 3. Determination of the recognition sequence for the specific cleavage of ceiE7-mRNA. (A) A schematic illustration of mutations used in this study. The mutated nucleotides generated by PCR-mediated site-directed mutagenesis are underlined in each mutant clone. The arrows denote the major $\left(G^{\nabla} A\right)$ and minor $\left(A^{\nabla} U\right)$ cleavage sites, respectively. (B) Gel electrophoresis of cDNAs derived from the primer extension. Total RNAs were extracted from E. coli cells containing the recombinant $c e i E 7$ gene with mutations flanking the cleavage sites. Portions of RNAs $(50 \mu \mathrm{g})$ were analyzed by primer extension. Expressions of ampicillin resistant gene encoded on these recombinant plasmids were exhibited as internal control. Lanes 1 and 2, samples from cells harboring $\mathrm{pSC} 02$ and $\mathrm{pSC} 02-14 \mathrm{R}$ were used as positive and negative control, respectively. The levels of specific cleavage signals visualized on gel were symbolized by "+" and "-" (A). The major and minor cleavage signals from cells containing pSC02 were denoted as "+++" and "+", respectively (B, lane 1). Symbol "-" denoted as basal level/or no specific cleavage signal.

the recognition sequence for this specific cleavage, the sequence flanking the specific cleavage sites, GAUCUG AUU (Asp52-Leu53-Ile54) was chosen as the target for the site-directed mutagenesis without disrupting the translation frame of cei gene (Fig. 3A). Total RNA extracts from cells harboring the mutants, pSC02-5, -12, -24 , and -13 , were analyzed by primer extension (Fig. 3B). Signals of specific mRNA cleavage in all mutants were reduced to various degrees (Fig. 3B). Mutation of GA to AU adjacent to the major cleavage site caused the reduction of major cleavage signal but the concomitant minor cleavage signal was enhanced (Fig. 3B, lane 3).
Surprisingly, conversion of A to G mutant upstream of the minor cleavage site abolished the major but not the minor cleavage signal (Fig. 3B, lane 4). Furthermore, we observed that only mutants with nucleotide changes between the major and minor cleavage sites abolished both cleavage signals (Fig. 3B, lanes 5 and 6). The result of Western blot showed that all clones produced equal amounts of $\operatorname{Im} 7$ (data not shown). Furthermore, it is worth noting that the specific cleavage of $c e i E 7-\mathrm{mRNA}$ could be reconstituted in vitro by one of the mutant $\operatorname{Im} 7$

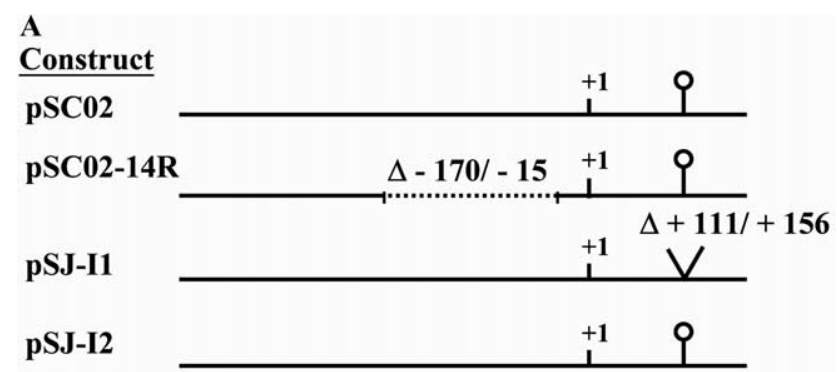

B



Fig. 4. Requirement of T1 stem-loop for the specific cleavage of the ceiE7-mRNA. Total RNAs ( $50 \mu \mathrm{g}$ for each) from cells harboring the ceiE7 genes with or without the downstream T1 stem-loop sequence were analyzed by primer extension. All clones used in this study were demonstrated in (A). pSC02, containing the wild-type ceiE7 gene, as the positive control; pSC02-14R, which part of ceiE7 coding region was deleted, as the negative control; pSJ-I1, the T1 stem-loop distal to the cei gene was deleted; and pSJ-I2, same as pSJ-I1 except that the T1 stemloop was reintroduced back to the downstream region of the ceiE7 gene. (B) Primer extension profiles of RNAs isolated from the clones described in (A). 5'-labeled oligonucleotide primer Pcei was used for all primer extension experiments. The product of primer extension of $a m p^{r}$ gene was used as internal control. Note that $a m p^{r}$ gene was not included in the constructions of recombinant plasmids pSJ-I1 and pSJ-I2. 
extracted from cells harboring pSC02-13 (data not shown). Thus, these results probably indicated that the mutant proteins still maintain its cleavage-related activity for the ceiE7-mRNA. Based on these observations we proposed that the recognition sequence for major and minor cleavages might be different, however, the internal sequence between the major and minor cleavage sites "-CAUU-" is essential for both cleavages.

\section{Secondary structure distal to the ceiE7-mRNA is essential for the specific cleavage}

It was found that an inverted repeat sequence (IRS) located between cei and cel genes of the ColE operon acts as a modulator for regulating the transcription readthrough from the SOS promoter to the downstream cel gene [29]. In addition, we reported that this IRS may also regulate the translation activity of the cel gene [8]. With the FOLDRNA computer prediction program from the GCG package, we predicated that this IRS may form a stem-loop structure [8]. Also, Bouvet and Belasco [30] proposed that determination of the position for specific cleavage by RNA endoribonuclease might be related to the secondary structure of the RNA. Thus, it is very interesting to test whether the predicted secondary structure (T1 stem-loop) of the ColE7 operon plays any role in the specific cleavage of the ceiE7-mRNA. In the mutant clone (pSJ-I1, see Fig. 4A), T1 stem-loop structure was deleted. Remarkably, the results of primer extension showed that the specific cleavage is abolished completely in cells harboring the mutant clone (Fig. 4B, lane 3). In contrast, when the T1 stem-loop structure was re-introduced back to the clone, the specific cleavage signal reappeared (pSJ-I2 was the same as $\mathrm{pSC} 02$ except that the cloning vector was pREP4 instead of pUC18, Fig. 4, lane 4). The mRNA structures from all tested mutants were predicted by computer folding using the MFOLD computer program from the GCG package based on the algorithm of Zuker and Stiegler [31] and FOLDRNA computer prediction program. The computer predication indicated that the overall structure of the ceiE7-mRNA coding region or stem-loop structure remained the same as the wild-type ceiE7-mRNA. Clearly, our experimental results demonstrated that the T1 stem-loop structure is essential for the activation of specific cleavage of ceiE7-mRNA.

It has been acknowledged that RNA folding or cleavage can be regulated by RNA binding proteins, which can be recruited to the target site by the presence of the loop region of the stem-loop structure of mRNA [32]. To elucidate the possible function of the loop region of the T1 stem-loop structure in this cleavage event, the original sequence of the loop region (130-UAAGG AUG-137) was converted to its complementary sequence (pSC02-10, Fig. 5A). Remarkably, the specific cleavage signal was not observed in cells harboring the
$\mathbf{A}$

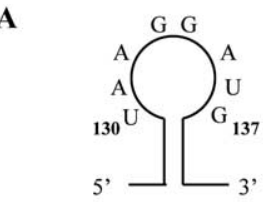

Construct pSC02

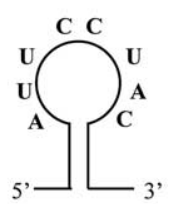

pSC02-10

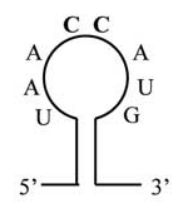

pSC02-25

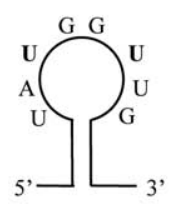

pSC02-26
B

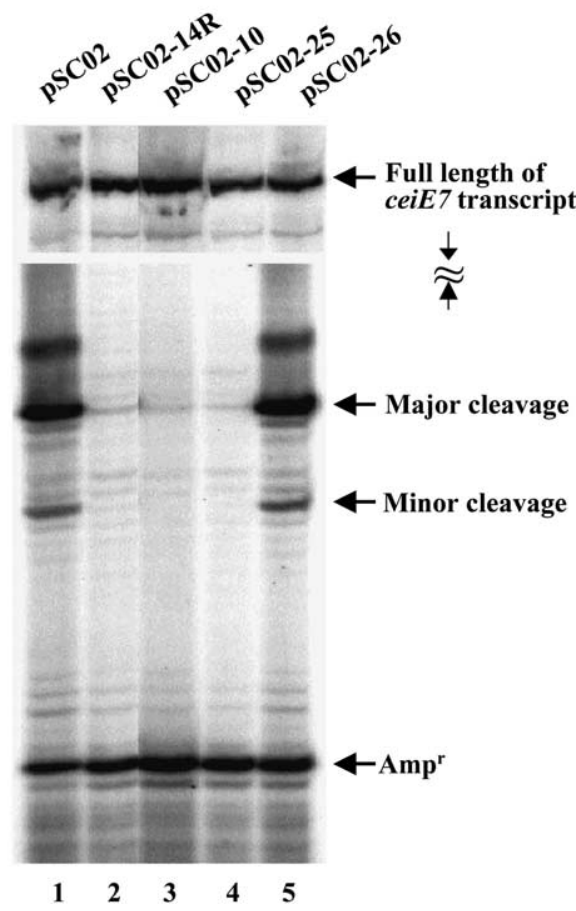

Fig. 5. Identification of critical nucleotide of the T1 stem-loop involved in the activation of the specific cleavage of $c e i E 7-m R N A$. (A) The wildtype and the mutant type sequences of the loop region of the T1 stemloop structure are shown. Recombinant plasmid pSC02 represents the wild-type loop sequence and the other three recombinant plasmids pSC02-10, -25 , and -26 are the three mutants used in this experiment. (B) Detection of the cleavage signals from cells harboring distinct mutant clones by primer extension. Lanes 1 and 2, pSC02 and pSC02$14 \mathrm{R}$, as positive and negative control, respectively; lane 3 , pSC02-10, the loop sequence was converted to the complementary sequence of original sequence; lane 4, pSC02-25, the topmost di-nucleotide $\mathrm{G}$ of the loop was converted to di-nucleotdie C; and lane 5, pSC02-26, the nucleotide $\mathrm{A}$ adjacent to the di-nucleotide $\mathrm{G}$ of the $\mathrm{T} 1$ stem-loop was converted to $\mathrm{U}$. The primer extension product of $a m p^{r}$ gene was used as internal control.

recombinant plasmid pSC02-10 (Fig. 5B, lane 3) when examined by primer extension. To locate the critical element of the loop region for the specific cleavage, a mutant pSC02-25 was constructed in which the di-nucleotide $\mathrm{GG}$ at the top position of the loop sequence was replaced by a CC di-nucleotide (Fig. 5A). Similarly, A$\mathrm{A}$ at 132 and $135 \mathrm{nt}$ was replaced by a TT di-nucleotide for the construction of pSC02-26 (Fig. 5A). Surprisingly, the specific cleavage signal was completely abolished in cells harboring pSC02-25 (Fig. 5B, lane 4) but not in cells with pSC02-26 (Fig. 5B, lane 5). These results clearly demonstrated that two consecutive GG 
nucleotides at the topmost region of the putative loop structure are essential for the specific cleavage of ceiE7mRNA.

\section{Discussion}

We have confirmed the fact that $\operatorname{Im} 7$ played a fundamental role for initiating the specific cleavage of ceiE7-mRNA both in vivo (Fig. 1) and in vitro (Fig. 2). In this report, we further demonstrated that the putative T1 stem-loop structure located between the cei and cel genes plays a dual role to initiate the specific cleavage of the ceiE7-mRNA as well as to regulate the translation initiation of the cel gene (our unpublished data). The GG di-nucleotide on the topmost position of the loop region of the putative $\mathrm{T} 1$ stem-loop structure might be crucial for the regulation of the ColE7 operon.

Site-specific endonucleolytic cleavage found in the untranslated region (UTR) of mRNAs is a universal strategy for the regulation of gene expression both in prokaryotes [33-36] and eukaryotes [37-39]. A recent report indicated that a conserved stem-loop structure of the rne $5^{\prime}$ UTR functions as a sensor in response to changes in cellular RNase E activity [32]. Thus, autoregulatory expression of genes modulated by specific cleavage at the UTR of mRNAs is of biological and environmental relevance in above cases. In contrast, the $\operatorname{Im} 7$-mediated specific cleavage is located at the coding region of its own cei-mRNA. We have already ruled out the possibility that RNase E and RNase III are involved in the specific cleavage of cei-mRNA [8]. Our finding is not unique, as Loomis and Moseley [40] also indicated that processing of a coding sequence of the F1845 fimbrial operon of E. coli is independent of RNase E and RNase III.

Interactions of protein-small RNA hairpin have been characterized in E. coli DEAD helicase (DbpA) with $23 \mathrm{~S}$ rRNA [41] as well as eukaryotic initiation factor 3 (elF3) with hepatitis $\mathrm{C}$ virus (HCV) internal ribosome entry site (IRES) RNA [42,43]. These interactions are essential for activating the helicase or mediate translation initiation. Thus, it is a plausible hypothesis that in the presence of the putative T1 stem-loop structure, the $\operatorname{Im} 7$ in conjunction with other protein components from the S-30 cell free extracts may direct a $3^{\prime}-5^{\prime}$ degradation of upstream mRNA, and at the same time play a part in the regulation of the translational expression of downstream cel gene (our unpublished data). Thus, the biological relevance of mRNA cleavage is unambiguous in modulating gene expression at the levels of mRNA stabilities.

The RNA degradation is a highly organized process performed by the complicated complexes such as the degradosome in prokaryotes [12] and the exosome in eukaryotes [44]. Our results suggest the existence of an alternative mRNA degradative machinery that allows the feedback involvement of the translation product from target mRNA. Therefore, to identify the protein complex from the S-30 cell free extracts for the specific cleavage of ceiE7-mRNA is of remarkable interest for the exploration of a novel RNA decay pathway.

\section{Acknowledgments}

This work was supported by research grants from the National Science Council of the Republic of China to K.-F. Chak (NSC 90-2320-010-089- and NSC 91-2320-B-010-043-).

\section{References}

[1] R. James, C. Kleanthous, G.R. Moore, The biology of E colicins: paradigms and paradoxes, Microbiology 142 (1996) 1569-1580.

[2] C.J. Lazdunski, E. Bouveret, A. Rigal, L. Journet, R. Lloubes, H. Benedetti, Colicin import into Escherichia coli cells, J. Bacteriol. 180 (1998) 4993-5002.

[3] K. Jakes, N.D. Zinder, T. Boon, Purification and properties of colicin E3 immunity protein, J. Biol. Chem. 249 (1974) 438444.

[4] C. Kleanthous, R. James, A.M. Hemmings, G.R. Moore, Protein antibiotics and their inhibitors, Biochem. Soc. Trans. 27 (1999) 63-67.

[5] T.P. Ko, C.C. Liao, W.Y. Ku, K.F. Chak, H.S. Yuan, The crystal structure of the DNase domain of colicin E7 in complex with its inhibitor Im7 protein, Struct. Fold. Des. 7 (1999) 91-102.

[6] A.P. Pugsley, M. Schwartz, Colicin E2 release: lysis, leakage or secretion? Possible role of a phospholipase, EMBO J. 3 (1984) 2393-2397.

[7] F.M. Lu, K.F. Chak, Two overlapping SOS-boxes in ColE operons are responsible for the viability of cells harboring the Col plasmid, Mol. Gen. Genet. 251 (1996) 407-411.

[8] S.Y. Hsieh, T.P. Ko, M.Y. Tseng, W. Ku, K.F. Chak, H.S. Yuan, A novel role of ImmE7 in the autoregulatory expression of the ColE7 operon and identification of possible RNase active sites in the crystal structure of dimeric ImmE7, EMBO J. 16 (1997) 1444 1454 .

[9] K.F. Chak, M.K. Safo, W.Y. Ku, S.Y. Hsieh, H.S. Yuan, The crystal structure of the immunity protein of colicin E7 suggests a possible colicin-interacting surface, Proc. Natl. Acad. Sci. USA 93 (1996) 6437-6442.

[10] T.E. Arnold, J. Yu, J.G. Belasco, mRNA stabilization by the ompA $5^{\prime}$ untranslated region: two protective elements hinder distinct pathways for mRNA degradation, RNA 4 (1998) 319330.

[11] M. Grunberg-Manago, Messenger RNA stability and its role in control of gene expression in bacteria and phages, Annu. Rev. Genet. 33 (1999) 193-227.

[12] R. Rauhut, G. Klug, mRNA degradation in bacteria, FEMS Microbiol. Rev. 23 (1999) 353-370.

[13] J. Belasco, G. Brawerman, Control of Messenger RNA Stability, Academic Press, New York, 1993.

[14] E.A. Mudd, C.F. Higgins, Escherichia coli endoribonuclease RNase E: autoregulation of expression and site-specific cleavage of mRNA, Mol. Microbiol. 9 (1993) 557-568.

[15] S.A. Emory, J.G. Belasco, The ompA $5^{\prime}$ untranslated RNA segment functions in Escherichia coli as a growth-rate-regulated mRNA stabilizer whose activity is unrelated to translational efficiency, J. Bacteriol. 172 (1990) 4472-4481. 
[16] C.P. Ehretsmann, A.J. Carpousis, H.M. Krisch, Specificity of Escherichia coli endoribonuclease RNase E: in vivo and in vitro analysis of mutants in a bacteriophage T4 mRNA processing site, Genes Dev. 6 (1992) 149-159.

[17] S.A. Emory, P. Bouvet, J.G. Belasco, A 5'-terminal stem-loop structure can stabilize mRNA in Escherichia coli, Genes Dev. 6 (1992) $135-148$.

[18] P. Alifano, C.B. Bruni, M.S. Carlomagno, Control of mRNA processing and decay in prokaryotes, Genetica 94 (1994) 157-172.

[19] R.S. McLaren, S.F. Newbury, G.S. Dance, H.C. Causton, C.F. Higgins, mRNA degradation by processive $3^{\prime}-5^{\prime}$ exoribonucleases in vitro and the implications for prokaryotic mRNA decay in vivo, J. Mol. Biol. 221 (1991) 81-95.

[20] E. Hajnsdorf, P. Régnier, E. coli $\operatorname{rps} O$ mRNA decay: RNase E processing at the beginning of the coding sequence stimulates poly(A)-dependent degradation of the mRNA, J. Mol. Biol. 286 (1999) 1033-1043.

[21] R.S. Cormack, G.A. Mackie, Structural requirements for the processing of Escherichia coli $5 \mathrm{~S}$ ribosomal RNA by RNase E in vitro, J. Mol. Biol. 228 (1992) 1078-1090.

[22] G.A. Mackie, J.L. Genereaux, The role of RNA structure in determining RNase E-dependent cleavage sites in the mRNA for ribosomal protein S20 in vitro, J. Mol. Biol. 234 (1993) 998-1012.

[23] S. Lin-Chao, T.T. Wong, K.J. McDowall, S.N. Cohen, Effects of nucleotide sequence on the specificity of rne-dependent and RNase E-mediated cleavages of RNA I encoded by the pBR322 plasmid, J. Biol. Chem. 269 (1994) 10797-10803.

[24] K.J. McDowall, S. Lin-Chao, S.N. Cohen, A + U content rather than a particular nucleotide order determines the specificity of RNase E cleavage, J. Biol. Chem. 269 (1994) 10790-10796.

[25] K.F. Chak, W.S. Kuo, F.M. Lu, R. James, Cloning and characterization of the ColE7 plasmid, J. Gen. Microbiol. 137 (1991) 91-100.

[26] J. Sambrook, E.F. Fritsch, T. Maniatis, Molecular Cloning: A Laboratory Manual, second ed., Cold Spring Harbor Laboratory Press, New York, 1989.

[27] A. von Gabain, J.G. Belasco, J.L. Schottel, A.C. Chang, S.N. Cohen, Decay of mRNA in Escherichia coli: investigation of the fate of specific segments of transcripts, Proc. Natl. Acad. Sci. USA 80 (1983) 653-657.

[28] G.A. Mackie, Specific endonucleolytic cleavage of the mRNA for ribosomal protein S20 of Escherichia coli requires the product of the ams gene in vivo and in vitro, J. Bacteriol. 173 (1991) 24882497.

[29] K.F. Chak, R. James, Analysis of the promoters for the two immunity genes present in the ColE3-CA38 plasmid using two new promoter probe vectors, Nucleic Acids Res. 13 (1985) 25192531.
[30] P. Bouvet, J.G. Belasco, Control of RNase E-mediated RNA degradation by $5^{\prime}$-terminal base pairing in E. coli, Nature 360 (1992) 488-491.

[31] M. Zuker, P. Stiegler, Optimal computer folding of large RNA sequences using thermodynamics and auxiliary information, Nucleic Acids Res. 9 (1981) 133-148.

[32] A. Diwa, A.L. Bricker, C. Jain, J.G. Belasco, An evolutionarily conserved RNA stem-loop functions as a sensor that directs feedback regulation of RNase E gene expression, Genes Dev. 14 (2000) 1249-1260.

[33] U. Schmeissner, K. McKenney, M. Rosenberg, D. Court, Removal of a terminator structure by RNA processing regulates int gene expression, J. Mol. Biol. 176 (1984) 39-53.

[34] T.C. King, R. Sirdeskmukh, D. Schlessinger, Nucleolytic processing of ribonucleic acid transcripts in prokaryotes, Microbiol. Rev. 50 (1986) 428-451.

[35] C. Portier, L. Dondon, M. Grunberg-Manago, P. Régnier, The first step in the functional inactivation of the Escherichia coli polynucleotide phosphorylase messenger is a ribonuclease III processing at the $5^{\prime}$ end, EMBO J. 6 (1987) 2165-2170.

[36] J.C. Bardwell, P. Régnier, S.M. Chen, Y. Nakamura, M. Grunberg-Manago, D.L. Court, Autoregulation of RNase III operon by mRNA processing, EMBO J. 8 (1989) 3401-3407.

[37] C.A. Beelman, R. Parker, Degradation of mRNA in eukaryotes, Cell 81 (1995) 179-183.

[38] J. Ross, mRNA stability in mammalian cells, Microbiol. Rev. 59 (1995) 423-450.

[39] A. Jacobson, S.W. Peltz, Interrelationships of the pathways of mRNA decay and translation in eukaryotic cells, Annu. Rev. Biochem. 65 (1996) 693-739.

[40] W.P. Loomis, S.L. Moseley, Translational control of mRNA processing in the F1845 fimbrial operon of Escherichia coli, Mol. Microbiol. 30 (1998) 843-853.

[41] C.A. Tsu, K. Kossen, O.C. Uhlenbeck, The Escherichia coli DEAD protein DbpA recognizes a small RNA hairpin in 23S rRNA, RNA (New York, NY) 7 (2001) 702-709.

[42] A.J. Collier, J. Gallego, R. Klinck, P.T. Cole, S.J. Harris, G.P. Harrison, F. Aboul_Ela, G. Varani, S. Walker, A conserved RNA structure within the HCV IRES eIF3-binding site, Nat. Struct. Biol. 9 (2002) 375-380.

[43] J.S. Kieft, K. Zhou, A. Grech, R. Jubin, J.A. Doudna, Crystal structure of an RNA tertiary domain essential to HCV IRESmediated translation initiation, Nat. Struct. Biol. 9 (2002) 370374.

[44] P. Mitchell, E. Petfalski, A. Shevchenko, M. Mann, D. Tollervey, The exosome: a conserved eukaryotic RNA processing complex containing multiple $3^{\prime} \rightarrow 5^{\prime}$ exoribonucleases, Cell 91 (1997) $457-$ 466. 